\title{
Some Advantages of the RDM-arithmetic of Intervally-Precisiated Values
}

\author{
Andrzej Piegat , Marcin Pluciński \\ Faculty of Computer Science and Information Technology, \\ West Pomeranian University of Technology, \\ Żotnierska 49, 71-210 Szczecin, Poland \\ E-mail: apiegat@wi.zut.edu.pl,mplucinski@wi.zut.edu.pl
}

Received 20 May 2015

Accepted 13 October 2015

\begin{abstract}
Moore's interval arithmetic always provides the same results of arithmetic operations, e.g. $[1,3]+[5,9]=$ $[6,12]$. But in real life problems, the operation result can be different, e.g. equal to [4,7]. Therefore, real problems require more advanced arithmetic. The paper presents (on example of the division) an arithmetic of intervally-precisiated values (IPV-arithmetic) and its main advantages. Thanks to it, it is possible to process different tasks that people solve intuitively. The most important advantages are: existence of inverse elements of addition and multiplication, holding the distributivity law and the cancellation law of multiplication, possibility of achieving not only the solution span $[\underline{x}, \bar{x}]$ but also the full, multidimensional solution and its cardinality distribution without using Monte Carlo method, possibility of achieving unique and complete solution sets of equations with unknowns, possibility of calculations with uncorrelated IPVs, possibility of calculations with fully correlated and partly correlated IPVs, possibility of uncertainty decreasing of original data items occurring in problems. All these advantages are illustrated and visualised by examples.
\end{abstract}

Keywords: Interval arithmetic, Interval-precisiation arithmetic, Granular computing, Interval equations, Computing with words

\section{Introduction}

An interval arithmetic defines basic operations on intervals: addition, subtraction, multiplication and division. Elaboration of its basic concepts and rules is assigned to R.E. Moore, who published his first book ${ }^{1}$ on this subject in 1966 and the latest ${ }^{2}$ in 2009. This arithmetic was called interval one, because with its use one can e.g. add two quantities $a$ and $b$, which values are not precisely but only approximately known and their approximation or the precisiation has a form of an interval, e.g. $a=[1,3]$ and $b=[3,5]$. The name interval arithmetic and other notions introduced by Moore (that are widely used in books about this arithmetic) are rather im- proper according to authors because they lead to a little incorrect understanding of this arithmetic and to incorrect approaches to calculation methods elaborated by many scientists. In books on interval arithmetic there are used such notions as an interval addition, an interval subtraction, etc. However, in reality we do not want to add intervals but add e.g. two not precisely known values $a$ and $b$, which were precisiated in the interval form on the basis of technical measurements or expert evaluations (the word precisiation was used by L. Zadeh in his publications on Computing with Words ${ }^{3}$ ).

As it will be shown, this new understanding of intervals has a great meaning and leads to a new arithmetic that is proposed to be 
called the arithmetic of intervally-precisiated values (shortly IPV-arithmetic) or alternatively the arithmetic of intervally-approximated values (IAVarithmetic). The IPV-arithmetic seems to be a less important area of mathematics and many scientists and engineers do not use it or even do not know about its existence. However, the IPV-arithmetic has become a very important branch of mathematics in consequence of awareness of the fact by many engineers and scientists that for achieving more credible and more informative problem solutions any available information piece about the problem should be used. Not only numerical and precise $(x=$ 1.7562883 ) but also all approximate data pieces as e.g. $y$ is small or $z \in[13,15]$ should be used. This aim is realised by a popular and rapidly developing Grey Systems Theory ${ }^{4}$, a theory that undoubtedly can become 'mathematics of the future'. A very similar aim also has Granular Computing ${ }^{5}$.

Interval precisiations are probably the most frequently precisiation forms used in practice. Any technical measurements or evaluations (human measurements) generated by human experts can be formulated as intervals and a width of such interval depends on the measuring device characteristic. In practice, all or almost all continuous variables, as e.g. temperature, are measured with an error. Thus, they never can be known precisely. Only discrete variables as e.g. sum of money in a wallet can be measured precisely. In scientific research, in engineering, in economy, in medicine and in other areas of human activity, mathematical models contain variables and coefficients. At present, usually only precise knowledge of variables and parameter values is assumed in problem solving. However, such calculated results often considerably differ from real observations. The reason of this state of matter is ignoring data uncertainty and applying in mathematical models only variables, which are known 'precisely' (though, frequently the precision is an illusion only). If variables, which values are known approximately, are not taken into account, then dimensionality of a model will be reduced and this reduction can result in great quantitative and qualitative errors (e.g. the real modelled system is nonlinear and its dimensionally reduced model is a linear one). In practice, most variables and model parameters are known only approximately so arithmetic of IP-values can have application almost everywhere.

As an example let us consider a model called Wenger-Bach formula ${ }^{6}$ describing a dependence between a ship-rudder angle $\delta\left[^{\circ}\right]$ and its rotational velocity $r[\%]$ at small deflections from a constant ship speed:

$$
\begin{aligned}
\ddot{r}(t)+\left(\frac{1}{T_{1}}+\frac{1}{T_{2}}\right) & \dot{r}(t)+\frac{1}{T_{1} T_{2}}\left(r^{3}(t)+r(t)\right) \\
& =\frac{K}{T_{1} T_{2}}\left(T_{3} \dot{\delta}(t)+\delta(t)\right) .
\end{aligned}
$$

Exemplary values of coefficients can be:

$$
\begin{array}{ll}
T_{1}=-1183.7[\mathrm{~s}], & T_{2}=31.5[\mathrm{~s}], \\
T_{3}=100.1[\mathrm{~s}], & K=0.084[1 / \mathrm{s}],
\end{array}
$$

and then the final model looks like:

$$
\begin{aligned}
\ddot{r}(t) & +3090.12 \cdot 10^{-5} \cdot \dot{r}(t)-2.68 \cdot 10^{-5} \cdot\left(r^{3}(t)+r(t)\right) \\
& =-23.02 \cdot 10^{-5} \cdot \dot{\delta}(t)-0.23 \cdot 10^{-5} \cdot \delta(t) .
\end{aligned}
$$

In the formula (1), $T_{1}, T_{2}, T_{3}, K$ are coefficients specific for the considered ship. Meaning of variables $r$ and $\delta$ is explained in Fig. 1.

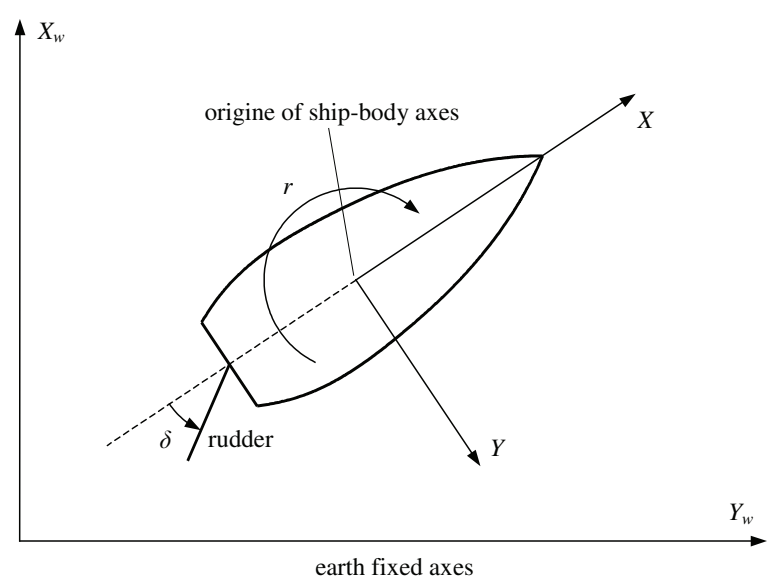

Fig. 1. Coordinate system of a ship movement, $\delta\left[^{\circ}\right]$ - rudder angle, $r[\% / s]$ - rotational speed of the ship

Coefficient values in the model (1) are constant numbers only then, when the ship is moving with 
a constant forward velocity and ship loading conditions (draught, longitudinal list and side list) and all other sea conditions (waves, stream, etc.) are identical as during ship identification experiments. If the above conditions are different, model coefficients will change their values. Thus, the ship model (1) can be called idealistic or academic or laboratory one, because the real, actual ship model always differs from it. Model coefficients vary in certain intervals in real conditions. Therefore, the realistic model (3) taking into account the coefficient variability should be used in the ship movement analysis.

$$
\begin{aligned}
\ddot{r}(t)+ & \left(\frac{1}{\left[\underline{T_{1}}, \overline{T_{1}}\right]}+\frac{1}{\left[\underline{T_{2}}, \overline{T_{2}}\right]}\right) \dot{r}(t) \\
& +\frac{1}{\left[\underline{T_{1}}, \overline{T_{1}}\right] \cdot\left[\underline{T_{2}}, \overline{T_{2}}\right]}\left(r^{3}(t)+r(t)\right) \\
= & \frac{[\underline{K}, \bar{K}]}{\left[\underline{T_{1}}, \overline{T_{1}}\right] \cdot\left[\underline{T_{2}}, \overline{T_{2}}\right]}\left(\left[\underline{T_{3}}, \overline{T_{3}}\right] \dot{\delta}(t)+\delta(t)\right)
\end{aligned}
$$

There can be given a large number of examples that illustrate the necessity of using mathematics of IP-values instead of the classical mathematics of precise numbers. Such mathematics is also necessary in various new science branches as e.g. an artificial intelligence.

An important area of the artificial intelligence is a fuzzy arithmetic $7,8,9$ in which an intervalbased calculation method called an $\alpha$-cut method is used. Next example is a probabilistic arithmetic $10,11,12$ in which operations on distributions require application of the interval arithmetic. The interval arithmetic has also been used in the case of word-models in methods of Computing with Words $3,13,14,15,16,17,18,19$. It is a very important branch of an artificial intelligence that conditions creation of the automatic thinking similar to the human one 20. The arithmetic of intervally-precisiated values is necessary for almost all problems with an uncertain, approximate information. However, at present the mostly used interval arithmetic type (examples can be books $2,4,7,21,22$ ) is first of all the Moore arithmetic $1,2,5$. This arithmetic can solve many problems and has found many applications ${ }^{23,24,25,26}$. It can be used anywhere where it is effective, however it has its own limitations. The consequence of this were attempts of improving it with different methods $21,27,28$.

Next, few drawbacks of the Moore arithmetic will be shortly presented.

1. The excess width effect.

2. The dependency problem.

3. Difficulties in solving of even simplest interval equations.

4. Problem of the interval equation's right-hand side.

5. Absurd solutions and request to introduce negative entropy into the system.

Because of the volume limitation of this paper, these drawbacks will not be discussed here - their explanation can be found for example in ${ }^{21,27,28}$ and in many other. In the next part of the paper there will be presented a multidimensional arithmetic of IP-values. Its concept was elaborated by Andrzej Piegat. Some basic concepts of this arithmetic were partly presented in $29,30,31,32,33,34,35$ and in this paper we will focus on a division of IPVs.

\section{Main concepts of the RDM-arithmetic}

If the precise value of a variable $x$ is not known but there is known an interval $[\underline{x}, \bar{x}]$ which contains this value, then a new variable $\alpha$ can be introduced that satisfies a condition $\alpha \in[0,1]$ and the original value $x$ can be expressed in the form:

$$
x=\underline{x}+\alpha_{x}(\bar{x}-\underline{x}), \quad \alpha_{x} \in[0,1] .
$$

An interval $X=[\underline{x}, \bar{x}]$ can be described as:

$$
X=\left\{x: x=\underline{x}+\alpha_{x}(\bar{x}-\underline{x}), \quad \alpha_{x} \in[0,1]\right\} .
$$

If e.g. $x \in[3,5]$ then such information can be expressed as:

$$
x=3+2 \alpha_{x}, \quad \alpha_{x} \in[0,1] .
$$

The $\alpha_{x}$ variable will be called RDM-variable (RDM: Relative-Distance-Measure). The interval 
notation is illustrated in Fig. 2.
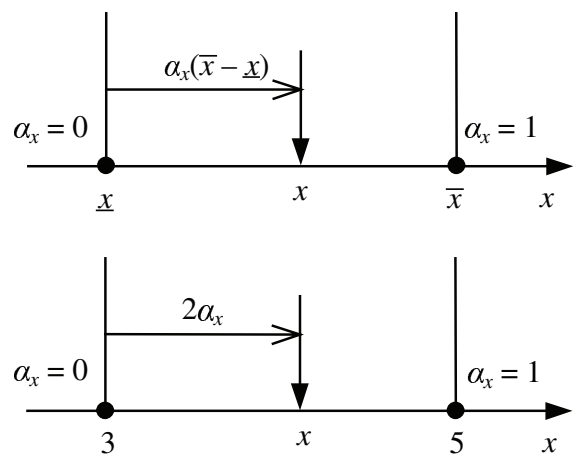

Fig. 2. Illustration of the meaning of the RDM-variable $\alpha_{x}$ in the case of a normal interval $[\underline{x}, \bar{x}], \bar{x}>\underline{x}$.

The aim of introducing RDM-variables is not to make unnecessary parametrisation of intervals but to introduce the Cartesian coordinate-system in the interval arithmetic, similarly as in the conventional crisp arithmetic, where it has been used since centuries. Thanks to the introduction of the RDMvariable an inside of the interval is not 'anonymous' and each value of the inside is determined by a corresponding value of the variable $\alpha$. Also, thanks to RDM-variables, the IPV-arithmetic becomes free from last 4 drawbacks of the Moore arithmetic quoted before.

The RDM-arithmetic has almost the same mathematical properties as the conventional arithmetic ${ }^{34}$. If $A, B, C$ are intervals, some of the most important properties of the RDM-arithmetic are listed in points 1-7.

1. $A+B=B+A, A B=B A-$ commutativity laws of addition and multiplication.

2. $A+(B+C)=(A+B)+C, A(B C)=(A B) C-$ associativity laws of addition and multiplication.

3. For each $A$ in $R$ there exists $-A$ in $R$ such that $A+(-A)=(-A)+A=0 .-A$ is the additive inverse of $A$.

4. $A(B+C)=A B+A C$ - left distributivity law, $(B+C) A=B A+C A-$ right distributivity law.

5. For each $A$ in $R, 0 \notin A$, there exists $A^{-1}=1 / A$ in $R$ such that $A A^{-1}=A(1 / A)=1 . A^{-1}$ is the multiplicative inverse of $A$.
6. $A+C=B+C \Rightarrow A=B$ - cancellation law of addition.

7. $C A=C B \Rightarrow A=B$ - cancellation law of multiplication.

In the case of the Moore's arithmetic, only first and second low hold ${ }^{2}$ ! It causes that transformations of formulas cannot be made and for this reason more complicated algebraic and mathematical problems cannot be solved.

It is very easy to prove that particular laws hold for the RDM-arithmetic. In formulas of the laws, RDM-models of intervals $A, B, C$ should be inserted and the laws equations should be examined ${ }^{34}$. For example, let's prove the left distributivity law:

$$
A(B+C)=A B+A C
$$

Proof. Let's describe intervals $A, B, C$ in the RDMnotation:

$$
\begin{aligned}
& A=\left\{a: a=\underline{a}+\alpha_{a}(\bar{a}-\underline{a}), \quad \alpha_{a} \in[0,1]\right\} . \\
& B=\left\{b: b=\underline{b}+\alpha_{b}(\bar{b}-\underline{b}), \quad \alpha_{b} \in[0,1]\right\} . \\
& C=\left\{c: c=\underline{c}+\alpha_{c}(\bar{c}-\underline{c}), \quad \alpha_{c} \in[0,1]\right\} . \\
A(B+C)=[\underline{a}, \bar{a}]([\underline{b}, \bar{b}]+[\underline{c}, \bar{c}]) & \\
= & \left\{a(b+c): a(b+c)=\left[\underline{a}+\alpha_{a}(\bar{a}-\underline{a})\right]\left[\underline{b}+\alpha_{b}(\bar{b}-\underline{b})\right.\right. \\
& \left.\left.+\underline{c}+\alpha_{c}(\bar{c}-\underline{c})\right], \alpha_{a}, \alpha_{b}, \alpha_{c} \in[0,1]\right\} \\
= & \left\{a(b+c): a(b+c)=\left[\underline{a}+\alpha_{a}(\bar{a}-\underline{a})\right]\left[\underline{b}+\alpha_{b}(\bar{b}-\underline{b})\right]\right. \\
& \left.+\left[\underline{a}+\alpha_{a}(\bar{a}-\underline{a})\right]\left[\underline{c}+\alpha_{c}(\bar{c}-\underline{c})\right], \alpha_{a}, \alpha_{b}, \alpha_{c} \in[0,1]\right\} \\
= & \left\{a b: a b=\left[\underline{a}+\alpha_{a}(\bar{a}-\underline{a})\right]\left[\underline{b}+\alpha_{b}(\bar{b}-\underline{b})\right], \alpha_{a}, \alpha_{b} \in[0,1]\right\} \\
& +\left\{a c: a c=\left[\underline{a}+\alpha_{a}(\bar{a}-\underline{a})\right]\left[\underline{c}+\alpha_{c}(\bar{c}-\underline{c})\right], \alpha_{a}, \alpha_{c} \in[0,1]\right\} \\
= & {[\underline{a}, \bar{a}][\underline{b}, \bar{b}]+[\underline{a}, \bar{a}][\underline{c}, \bar{c}]=A B+B C }
\end{aligned}
$$

The distributive law holds because the interval $A$ is described with the same RDM-variable in both components on the right side of the law equation.

The main advantages of the RDM-arithmetic ale listed below.

(a) Complicated problems can be solved, thanks to possibility of an equation's transformation.

(b) Almost all laws of the arithmetic of crisp numbers hold for the RDM-arithmetic. 
(c) The RDM-arithmetic provides complete, multidimensional problem-solutions from which various simplified representations as a cardinality distribution, a span of solution (Moore's solution), a center of gravity can be derived.

(d) In the RDM-arithmetic, it is possible to take into account dependencies between intervals. In real problems they are very common. Such dependencies are easily described by RDM-variables.

Last two advantages can be illustrated by a following example. In the classic interval arithmetic, as a result of subtracting two identical intervals we have:

$$
Y=X-X=[\underline{x}, \bar{x}]-[\underline{x}, \bar{x}]=[\underline{x}-\bar{x}, \bar{x}-\underline{x}] .
$$

If $A=[1,4]$ :

$$
A-A=[1,4]-[1,4]=[-3,3] .
$$

In the RDM-arithmetic, we can introduce two RDM-variables $\alpha_{x 1}, \alpha_{x 2} \in[0,1]$ :

$$
\begin{aligned}
Y & =X-X=\left(\underline{x}+\alpha_{x 1}(\bar{x}-\underline{x})\right)-\left(\underline{x}+\alpha_{x 2}(\bar{x}-\underline{x})\right) \\
& =\left(\alpha_{x 1}-\alpha_{x 2}\right)(\bar{x}-\underline{x}) .
\end{aligned}
$$

The result is a function of two RDM-variables $\alpha_{x 1}$ and $\alpha_{x 2}: Y=f\left(\alpha_{x 1}, \alpha_{x 2}\right)$. We can calculate from it many parameters, eg. an interval lower and upper bound. The function value is the smallest for $\alpha_{x 1}=0$ and $\alpha_{x 2}=1-$ in that way we can get the lower bound of the resulting interval which is equal $\underline{x}-\bar{x}$. The function value is the greatest for $\alpha_{x 1}=1$ and $\alpha_{x 2}=0$ - in that way we can get the upper bound of the resulting interval which is equal $\bar{x}-\underline{x}$.

If, however, we know that in the formula we have the same interval $X$, we can assume that $\alpha_{x 1}=\alpha_{x 2}=$ $\alpha_{x}$. Then:

$Y=X-X=\left(\underline{x}+\alpha_{x}(\bar{x}-\underline{x})\right)-\left(\underline{x}+\alpha_{x}(\bar{x}-\underline{x})\right)=0$,

what is consistent with common sense, but unattainable for the classic interval arithmetic.

All advantages of the RDM-arithmetic are illustrated in examples in next sections. Examples in the paper are mainly focused on the division of IPV's.

\section{Division of intervally-precisiated values}

According to the Moore arithmetic, a division of intervals $A / B=X$ should be realised with the formula (9) that is based on and results from a multiplication of intervals (10).

$$
[\underline{a}, \bar{a}] /[\underline{b}, \bar{b}]=[\underline{a}, \bar{a}] \cdot[1 / \bar{b}, 1 / \underline{b}], \quad 0 \notin[b]
$$

If we denote $1 / \bar{b}=\underline{d}$ and $1 / \underline{b}=\bar{d}$ then the formula (10) can be used for the division.

$$
\begin{aligned}
{[\underline{a}, \bar{a}] \cdot[\underline{d}, \bar{d}]=} & {[\min \{\underline{a} \cdot \underline{d}, \underline{a} \cdot \bar{d}, \bar{a} \cdot \underline{d}, \bar{a} \cdot \bar{d}\},} \\
& \max \{\underline{a} \cdot \underline{d}, \underline{a} \cdot \bar{d}, \bar{a} \cdot \underline{d}, \bar{a} \cdot \bar{d}\}]
\end{aligned}
$$

Fig. 3 presents a functional surface of the division of IP-values $a$ and $b: a / b=x$. Contour lines of constant values of division results $a / b=x=$ const can be projected from a $3 \mathrm{D}$-space onto a $2 \mathrm{D}$-space $A \times B$, what is shown in Fig. 4 .

Let us notice that the functional surface of the division, Fig. 3, consists on an infinite number of point solutions in form of triplets $\{a, b, x\}, x=a / b$. The 'solution' (9) and (10) provided by the Moore arithmetic is only information about the widest interval $[\underline{x}, \bar{x}]$ contained in the full, 3-dimensional solution set shown in Fig. 3. Thus, the Moore 'solution' can be called a representation of the full result of the division of two IP-values $a$ and $b$.

The RDM-arithmetic interprets the division of two intervals $A=[a]$ and $B=[b]$ as the division of two crisp but only approximately known values $a$ and $b$, where $a \in[\underline{a}, \bar{a}]$ and $b \in[\underline{b}, \bar{b}]$. Values $a$ and $b$ can be fully independent, partially dependent (e.g. $b \geqslant a$ ) or fully dependent (e.g. $a=b, a+b=1$, etc.). Generally a dependence of $a$ and $b$ means that a restriction $\mathbf{R}$ is imposed on pairs $(a, b)$ describing which pairs (tuples) are allowed to occur. Approximately known IP-values $a$ and $b$ are described with use of RDM-variables $\alpha_{a}$ and $\alpha_{b}$, so to any restriction $\mathbf{R}(a, b)$ corresponds a restriction $\mathbf{R}\left(\alpha_{a}, \alpha_{b}\right)$ expressed in terms of RDM-variables.

Definition of the division of intervals $A=[a]$ and $B=[b]$ under restriction $\mathbf{R}$, which corresponds to division of approximately known values $a$ and $b$, is expressed by (11). 


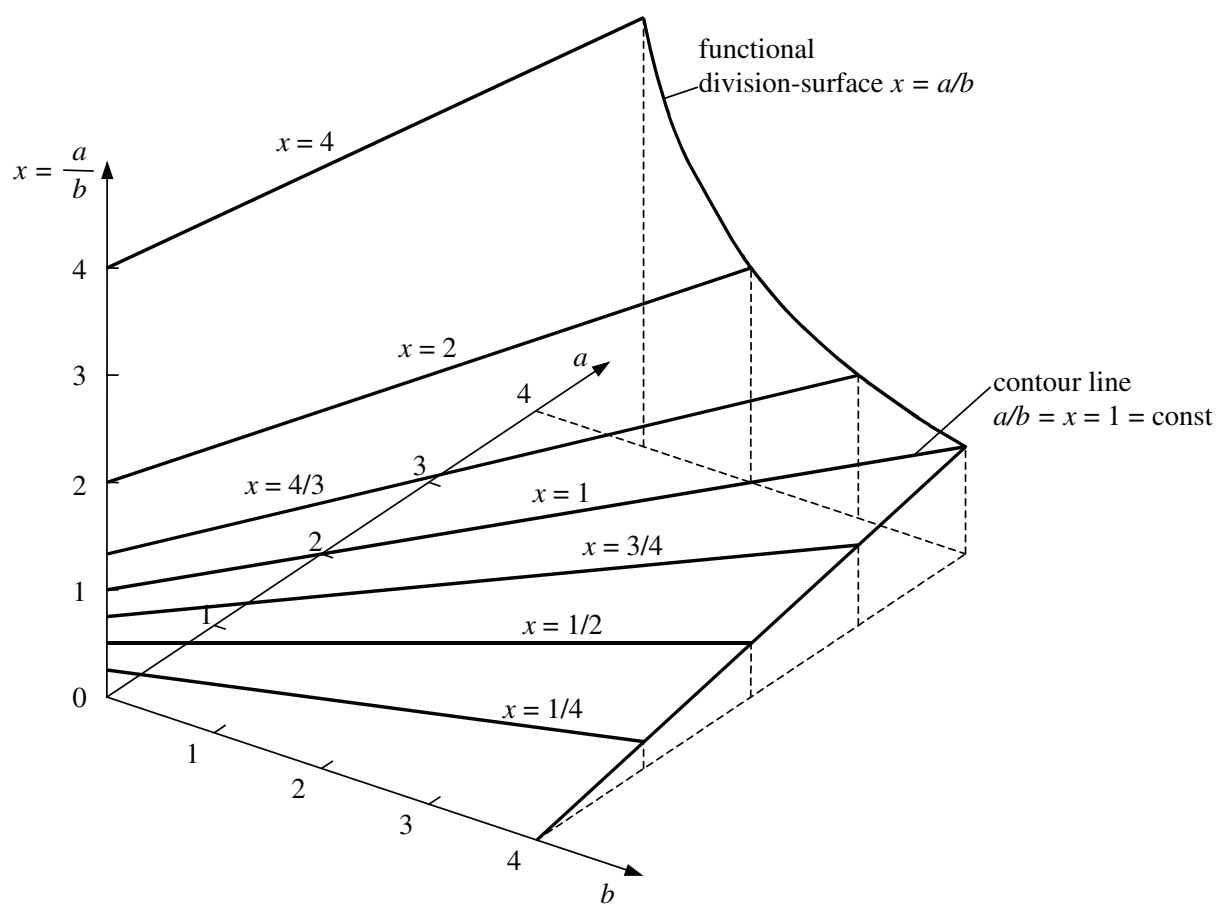

Fig. 3. Functional surface of the division $a / b=x$ for $a>0$ and $b>0$, where $x$ means a division result.

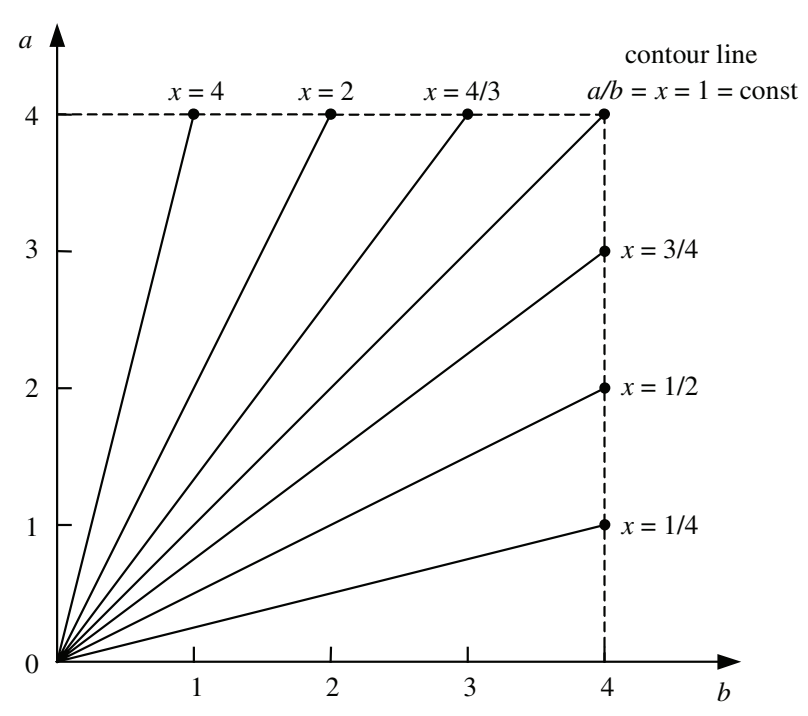

Fig. 4. Contour lines of constant values of the quotient $a / b=x$ in the $2 \mathrm{D}$-space $A \times B$.

$$
\begin{aligned}
& \frac{A}{B}=X: \frac{a}{b}=x=\frac{\underline{a}+(\bar{a}-\underline{a}) \alpha_{a}}{\underline{b}+(\bar{b}-\underline{b}) \alpha_{b}}, \\
& \mathbf{R} \quad \mathbf{R}(a, b) \quad \begin{array}{l}
\mathbf{R}\left(\alpha_{a}, \alpha_{b}\right) \\
\alpha_{a}, \alpha_{b} \in[0,1]
\end{array}
\end{aligned}
$$

Let us notice here, that formula (11) is 3dimensional (variables: $x, \alpha_{a}, \alpha_{b}$ ) which corresponds to the 3-dimensional division surface shown in Fig. 3. Formula (11) shows that in the RDM version of the division not only borders $\underline{a}, \bar{a}, \underline{b}, \bar{b}$ are taken into account (as in the Moore version) but also insides of intervals. It is possible thanks to continuous RDM-variables $\alpha_{a}$ and $\alpha_{b}$ and it extends possibilities of the interval arithmetic. It is also very important, that the Moore representation $[\underline{x}, \bar{x}]$ can be easily determined from simple examination of function (11) which will be shown further on.

Division can concern not only two intervals $A$ and $B$ but also three intervals: $A /(B C)$ or more. In this case, 3 RDM-variables $\alpha_{a}, \alpha_{b}, \alpha_{c}$ are used and the division formula (11) extends to (12).

$$
\frac{A}{B C}=X: \frac{a}{\mathbf{R}}=x=\frac{\underline{a}+(\bar{a}-\underline{a}) \alpha_{a}}{\mathbf{R}(a, b, c)} \underset{\left[\underline{b}+(\bar{b}-\underline{b}) \alpha_{b}\right]\left[\underline{c}+(\bar{c}-\underline{c}) \alpha_{c}\right]}{\mathbf{R}\left(\alpha_{a}, \alpha_{b}, \alpha_{c}\right)},
$$

$\alpha_{a}, \alpha_{b}, \alpha_{c} \in[0,1]$

The division result (12) exists in 4-dimensional space $\alpha_{a} \times \alpha_{b} \times \alpha_{c} \times X$. If the partial result $X_{1}=$ 
$A / B$ is known, then it can be used for calculation of the final result $X_{2}=A /(B C)$ according to formula (13).

$$
\frac{A}{B C}=\left(\frac{A}{B}\right) / C=\frac{X_{1}}{C}=X_{2}
$$

On the basis of the 4-dimensional result (13) its simplified representations, as e.g. the span $\left[\underline{x_{2}}, \overline{x_{2}}\right]$ (1-dimensional representation) or cardinality distribution can be determined.

There is a convention of denoting intervals by capital letters in the interval arithmetic literature ${ }^{2}$. In examples presented in the next part of the paper, small and capital letters will be used for various problem variables (IP-values) and intervals will be denoted in brackets: $[x]$.

Now, let us consider an example of the IPVs division: $a / b=x$.

Example 1: Possibility of achieving not only the solution span $[\underline{x}, \bar{x}]$ but also the full solution and its carddinality distribution.

A truck driver has travelled distance $d[\mathrm{~km}]$ from City 1 to City 2 several times. Real travel distances have been one time shorter, other time longer because of road repairs and detours. Generally, the travelled distance has been in the interval $d \in[750,800] \mathrm{km}$. The average travel speed also has changed and has been in the interval $v \in$ $[53.333,62.500] \mathrm{km} / \mathrm{h}$. How great has been travel time $x[\mathrm{~h}]$ ?

The problem can be expressed in the form of equation:

$$
\frac{[d]}{[v]}=\frac{[\underline{d}, \bar{d}]}{[\underline{v}, \bar{v}]}=\frac{[750,800]}{[53.333,62.5]}=[\underline{x}, \bar{x}] .
$$

Equation (14) can be transformed with use of the Moore arithmetic into:

$$
\begin{aligned}
\frac{[d]}{[v]} & =\frac{[750,800]}{[53.333,62.5]}=[750,800] \cdot[0.016,0.01875] \\
& =[12,15]=[\underline{x}, \bar{x}]
\end{aligned}
$$

Solution (15) obtained by the Moore arithmetic is 1-dimensional and therefore its informative value is limited. Now, let us solve this problem with the
RDM-arithmetic. RDM-variables $\alpha_{d}$ and $\alpha_{v}$ are introduced in (16). Travel time, as variable we are looking for, is denoted by $x$.

$$
\begin{array}{rr}
d=750+50 \alpha_{d}, & \alpha_{d} \in[0,1] \\
v=53.333+7.1667 \alpha_{v}, & \alpha_{v} \in[0,1] \\
\frac{d}{v}= & \frac{d}{\underline{v}+\alpha_{d}(\bar{d}-\underline{d})}=\frac{750+50 \alpha_{d}(\bar{v}-\underline{v})}{53.333+7.167 \alpha_{v}}
\end{array}
$$

Variables $d$ and $v$ are independent, so no restrictions $\mathbf{R}$ on pairs $(d, v)$ are imposed.

Values of variables $d, v, x$ for various border values of $\alpha_{d}$ and $\alpha_{v}$ are given in Table 1. The problem of the IPVs division $d / v=x$ is presented in the 2D-space $D \times V$ in Fig. 5. Let us notice that the solution $d / v(16)$ is a 3-dimensional set of triplets $\left\{\alpha_{d}, \alpha_{v}, x\right\}, x=d / v$.

Table 1. Values of variables $d, v$ and $x=T$ for various border values of RDM-variables $\alpha_{d} \in[0,1]$ and $\alpha_{v} \in[0,1]$ where $x$ means the travel time between City 1 and City 2 .

\begin{tabular}{c|cccc}
\hline$\alpha_{d}$ & 0 & 0 & 1 & 1 \\
$\alpha_{v}$ & 0 & 1 & 0 & 1 \\
\hline$d$ & $\underline{d}=750$ & $\underline{d}=750$ & $\bar{d}=800$ & $\bar{d}=800$ \\
$v$ & $\underline{v}=53.33$ & $\bar{v}=62.5$ & $\underline{v}=53.33$ & $\bar{v}=62.5$ \\
\hline$x=T$ & $\underline{d} / \underline{v}=14.06$ & $\underline{d} / \bar{v}=12$ & $\bar{d} / \underline{v}=15$ & $\bar{d} / \bar{v}=12.8$ \\
\hline
\end{tabular}

The result obtained by the Moore arithmetic $x \in$ $[12,15]$ is not the exact result of the division $d / v=x$ that is given by (16) and shown in Fig. 5. It is only the widest interval contained in the exact multidimensional result (16). It can be called a representation of the exact result. Other representation can be a cardinality distribution $\operatorname{card}(x)$ of particular possible values $x=d / v$. Lengths $L_{i}$ of contour lines $x=$ const can be interpreted as occurrence cardinalities $\operatorname{card}(x)$ of particular values of $x^{31}$.

Caution! Contour lines of the division are not parallel - see Fig. 4. The length $L_{1}$ of the first contour line from Fig. 5 is determined by formula (17) and the second line $L_{2}$ by formula (18). In the considered case, lengths of contour lines are almost equal. Fig. 6 presents the normalised cardinality distribution $\operatorname{card}(x)$ of the division result of two IPVs $d / v=x$ from Example 1 . 


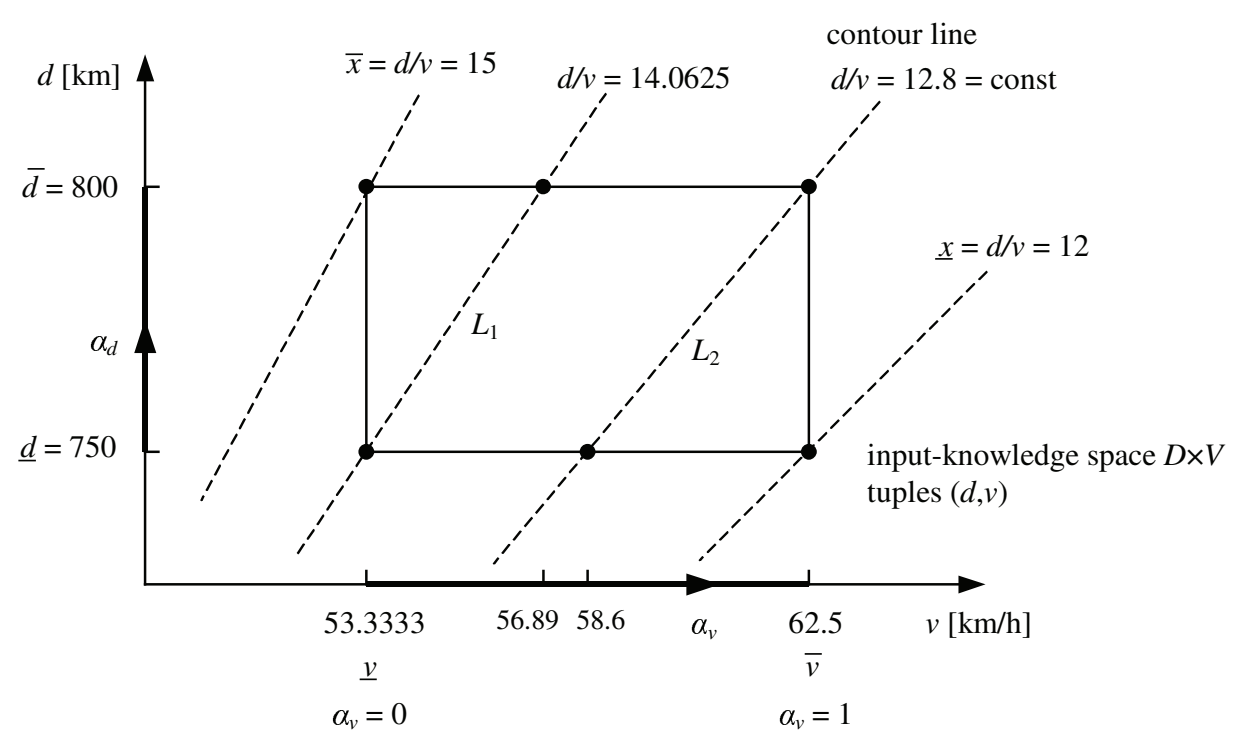

Fig. 5. Illustration of the IPVs division $d / v=x$ with use of the RDM-arithmetic, $\alpha_{d} \in[0,1], \alpha_{v} \in[0,1]$.

$$
\begin{aligned}
& L_{1}=\sqrt{(\bar{d}-\underline{d})^{2}+(\underline{v} \bar{d} / \underline{d}-\underline{v})^{2}} \\
& L_{2}=\sqrt{(\bar{d}-\underline{d})^{2}+(\bar{v}-\underline{d} \bar{v} / \bar{d})^{2}}
\end{aligned}
$$

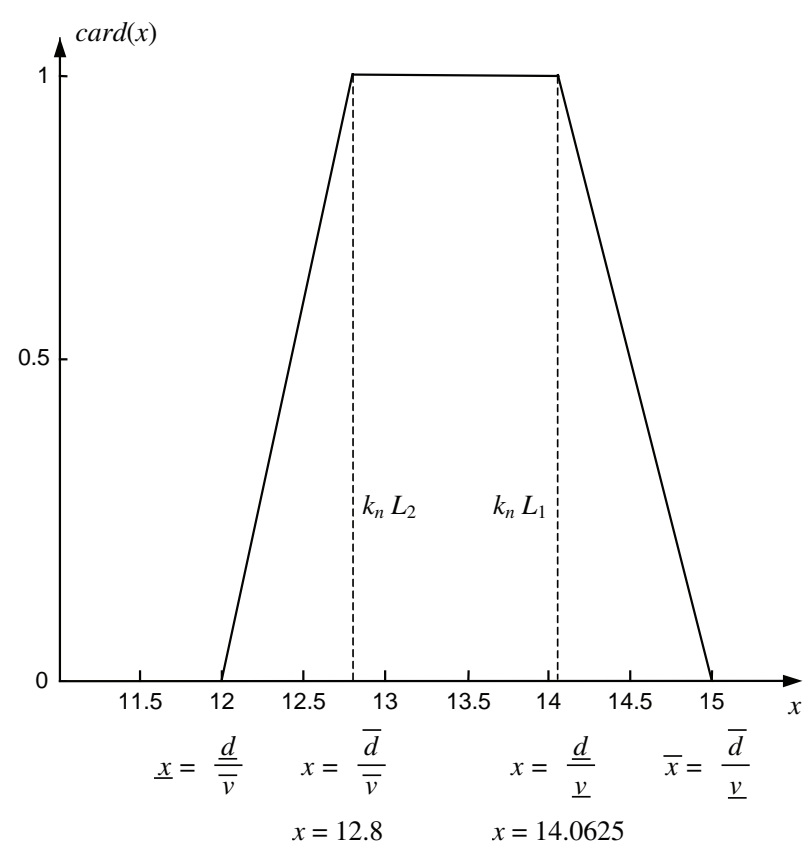

Fig. 6. Distribution of the normalised cardinality $\operatorname{card}(x)$ of possible results $x \in[\underline{x}, \bar{x}]=[12,15]$ of the IPVs division $d / v$ from Example 1, $k_{n}$ - normalising coefficient.

\section{Summary of the example.}

Results provided by the Moore arithmetic and the RDM-arithmetic can seem identical. However, this is not true. The Moore arithmetic doesn't distinguish that one and the same value of the division result $x$ can be generated by different tuples $(d, v)$. E.g. the value $x=12.8$ can be generated by $d / v=750 / 58.6$ or by $d / v=800 / 62.5$ and by many other tuples. It can be seen in Fig. 5. Because of this, the Moore arithmetic generates only the information about the widest interval $[12,15]$ contained in the full solution (16) - especially it can not produce such distributions of the cardinality $\operatorname{card}(x)$ as shown in Fig. 6. Such distributions can have a great meaning in the case of complex problems that are modelled by complicated mathematical formulas or by complicated schemes of information processing, e.g. Gantt schemes, PERT-schemes, etc.

Now, let us consider a backward calculation problem in the case of the interval division presented by Example 2.

Example 2: Possibility of achieving unique and complete solution sets of equations with unknowns.

A driver has travelled distance $d$ from City 1 to City 2 several times. His route has been one time shorter, other way longer: $d \in[750,800] \mathrm{km}$ because of road repairs and detours. Travel times also have 
varied: $T \in[12,15]$ h. How great has been the car velocity $x[\mathrm{~km} / \mathrm{h}]$ ?

Equation (19) is to be solved to find an answer to this question.

$$
\frac{[d]}{[x]}=[T], \quad \frac{[750,800]}{[\underline{x}, \bar{x}]}=[12,15]
$$

Solving this equation with the Moore arithmetic we can obtain the representation being the widest interval contained in the exact solution.

$$
[\underline{x}, \bar{x}]=[50.000,66.667]
$$

Now, let us see what result we obtain with the RDM-arithmetic. The variable $d$ can be expressed with use of the RDM-variable $\alpha_{d} \in[0,1]$ and the variable $T$ with use of $\alpha_{T} \in[0,1]$ :

$$
\begin{aligned}
& d=\underline{d}+\alpha_{d}(\bar{d}-\underline{d})=750+50 \alpha_{d}, \\
& T=\underline{T}+\alpha_{T}(\bar{T}-\underline{T})=53.333+9.167 \alpha_{T}, \\
& \alpha_{d} \in[0,1], \alpha_{T} \in[0,1] .
\end{aligned}
$$

Equation (19) can be also expressed with use of RDM-variables as:

$$
\begin{gathered}
\frac{\underline{d}+\alpha_{d}(\bar{d}-\underline{d})}{x}=\underline{T}+\alpha_{T}(\bar{T}-\underline{T}), \\
x=\frac{\underline{d}+\alpha_{d}(\bar{d}-\underline{d})}{\underline{T}+\alpha_{T}(\bar{T}-\underline{T})}=\frac{750+50 \alpha_{d}}{53.333+9.167 \alpha_{T}}, \\
\alpha_{d} \in[0,1], \alpha_{T} \in[0,1] .
\end{gathered}
$$

Equation (20) is the full and exact, multidimensional solution of the problem. Tab. 2 shows values of variables $d, T$ and $x=d / T$ for various border values of RDM-variables $\alpha_{d}$ and $\alpha_{T}$.

Table 2. Values of variables $d, x, T$ for various border values of RDM-variables $\alpha_{d}$ and $\alpha_{T}$.

\begin{tabular}{c|cccc}
\hline$\alpha_{d}$ & 0 & 0 & 1 & 1 \\
$\alpha_{T}$ & 0 & 1 & 0 & 1 \\
\hline$d$ & $\underline{d}=750$ & $\underline{d}=750$ & $\bar{d}=800$ & $\bar{d}=800$ \\
$T$ & $\underline{T}=12$ & $\bar{T}=15$ & $\underline{T}=12$ & $\bar{T}=15$ \\
\hline$x=d / T$ & $\underline{d} / \underline{T}=62.5$ & $\underline{d} / \bar{T}=50$ & $\bar{d} / \underline{T}=66.67$ & $\bar{d} / \bar{T}=53.33$ \\
\hline
\end{tabular}

Fig. 7 shows the 3D-illustration of the problem solution (20). The solution granule of the problem is more precisely shown in the 2D-space $(D \times X)$ in Fig. 8.
Fig. 8 shows the difference between the solution representation obtained by the Moore arithmetic $[\underline{x}, \bar{x}]=[50.000,66.667]$ and the solution of the RDM-arithmetic, where the solution is the set $\{(d, x)\}$ of tuples $(d, x)$ which satisfy conditions (20) or (21).

$$
\begin{aligned}
(d, v): \quad & d=750+50 \alpha_{d}, \quad \alpha_{d} \in[0,1] \\
& x \geqslant d / 15, \quad x \leqslant d / 12
\end{aligned}
$$

Fig. 8 clearly shows an imprecision of the Moore representation $[\underline{x}, \bar{x}]=[50.000,66.667]$ in relation to the full solution obtained by the RDM-arithmetic. No 1-dimensional representation of type $[\underline{x}, \bar{x}]$ provide such information as the solution obtained by the RDM-method. In case of doubts, whether the RDM-solution is really correct, it can be checked with the method of testing points. The point TP1 in Fig. 8 has coordinates $(d, x)=(800,50)$. It can be easily checked on the basis of the original equation $[750,800] /[\underline{x}, \bar{x}]=[12,15]$ that the quotient $d / x=$ $800 / 50=16$ is not contained in the interval $[12,15]$. Similarly, the test point $\operatorname{TP} 2(d, x)=(750,50)$ can be checked, Fig. 8 . It can be easily seen that the value of the quotient $d / x=750 / 50=15$ lies on the right edge of the interval $[12,15]$ of the considered equation.

A very interesting problem is the division of identical IPVs. It will be considered in Section 4.

\section{Division of 'identical' intervally-precisiated values}

If we divide two identical numbers using a classic arithmetic, we always obtain a result equal to 1 independently what specific problem we solve.

$$
\frac{3}{3}=\frac{5}{5}=\frac{-7}{-7}=\frac{a}{a}=1
$$

Dividing two same intervals with an application of the Moore arithmetic, we always obtain a certain interval including 1 as a result, and it is never a degenerated interval equal to $[1,1]$, for example:

$$
\frac{[A]}{[A]}=\frac{[1,3]}{[1,3]}=\left[\frac{1}{3}, 3\right] \text {. }
$$

Using the Moore arithmetic, independently what specific problem we solve, we will always obtain the 


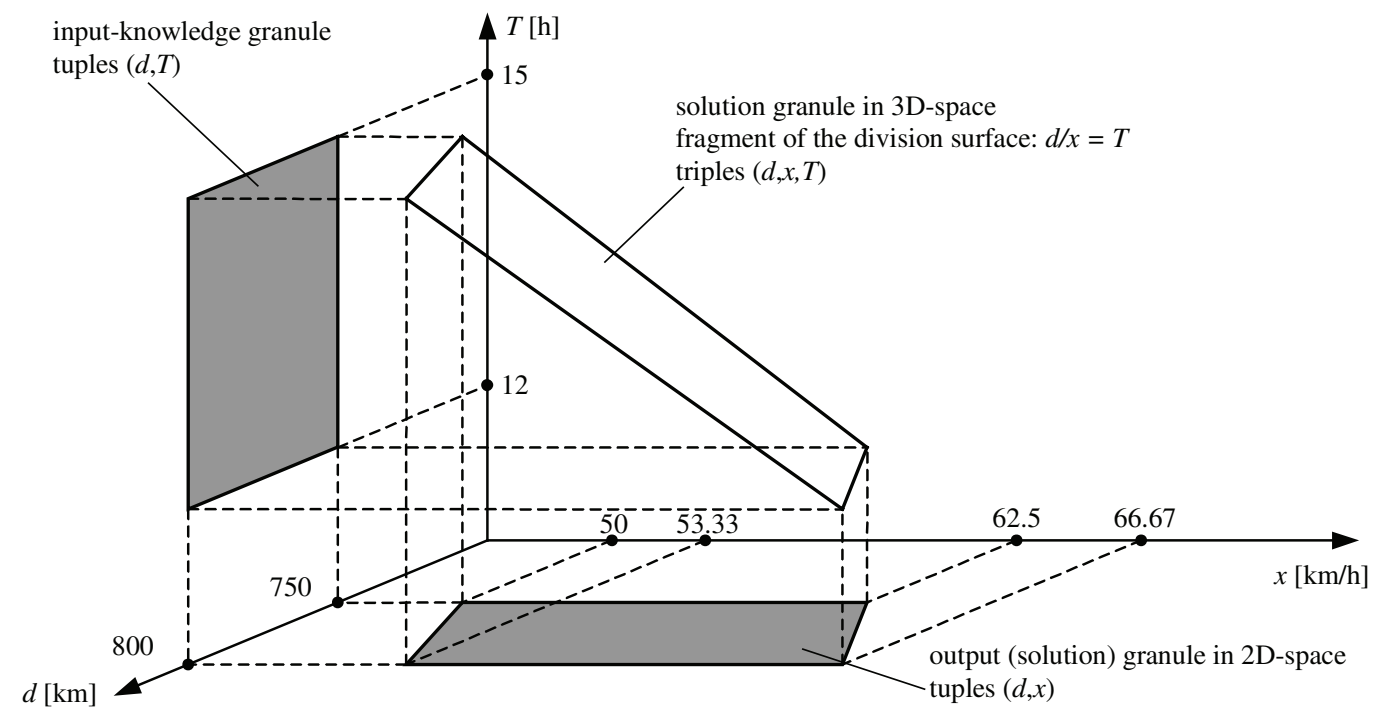

Fig. 7. Visualization of the IPVs division operation $d / x=T$ (backward-calculation type) in the 3D-space $D \times X \times T$.

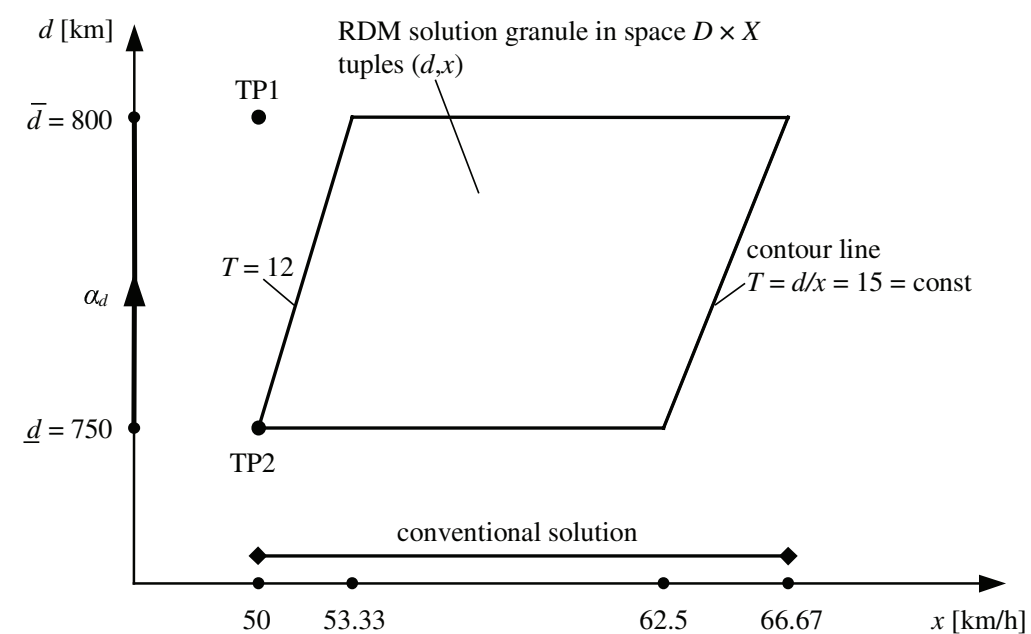

Fig. 8. Nonrectangular solution granule of the equation $d / x=T$ in the 2D-space $(D \times X)$; TP1 and TP2 - test points. 
same result $[1 / 3,3]$. It means, that like in a classic mathematics a general formula for interval dividing exists, as equations (9) and (10). Instead, the RDMarithmetic shows that an infinite number of results can be obtained from division of same IPVs! It is very astonishing and inconsistent with an intuition. Let's consider 3 following examples of dividing of same IPVs $[9,11] /[9,11]$ occurring in different problems. It confirms that operations on uncertain data are more complicated than we thought.

Example 3: Possibility of calculations with uncoupled (uncorrelated) IPVs.

A container with an irregular shape contains a material which volume $V\left[\mathrm{~m}^{3}\right]$ is evaluated as $V \in[9,11]$ $\mathrm{m}^{3}$. A specific volume of this material $V_{s p}\left[\mathrm{~m}^{3} /\right.$ ton] depends on a humidity degree and varies in the range $V_{s p} \in[9,11] \mathrm{m}^{3} /$ ton. The material is hard and its specific volume doesn't depend on its volume contained in the container. What is the weight $W$ [ton] of the material?

In the case when the material volume $V$ and the specific volume $V_{s p}$ is known with a perfect precision, the material weight can be calculated with an idealistic equation (24).

$$
W=\frac{V}{V_{s p}}
$$

Because our knowledge is approximate, the Moore arithmetic can be applied and for positive IPVs we achieve the widest interval (25).

$$
[\underline{W}, \bar{W}]=\frac{[\underline{V}, \bar{V}]}{\left[\underline{\left.V_{s p}, \overline{V_{s p}}\right]}\right.}=\frac{[9,11]}{[9,11]}=\left[\frac{9}{11}, \frac{11}{9}\right] .
$$

Now, let's apply the RDM-arithmetic to solve above problem. The volume $V$ of the material is known only approximately so it can be represented with the RDM-variable $\alpha_{V} \in[0,1]$ :

$$
V=\underline{V}+\alpha_{V}(\underline{V}-\bar{V})=9+2 \alpha_{V}, \quad \alpha_{V} \in[0,1] .
$$

The specific volume $V_{s p}$ is also known only approximately and it can be represented with the RDM-variable $\alpha_{V_{s p}} \in[0,1]$. Values of $V\left[\mathrm{~m}^{3}\right]$ and $V_{s p}\left[\mathrm{~m}^{3} /\right.$ ton $]$ are different in the general case although they are located in the same interval $[9,11]$.
A probability of an event that both variables are equal (for example $V=9.783265831 \ldots$ and $V_{s p}=$ $9.783265831 \ldots$ ) is infinitely small. As it was given in the problem description, the specific volume $V_{s p}$ doesn't depend on the volume $V$ of the material in the container, hence RDM-variables are different: $\alpha_{V} \neq \alpha_{V_{s p}}$.

The weight $W$ [ton] can be calculated from the formula:

$$
W=\frac{9+2 \alpha_{V}}{9+2 \alpha_{V_{s p}}}, \quad \alpha_{V} \in[0,1], \alpha_{V_{s p}} \in[0,1] .
$$

Equation (27) expresses the exact, multidimensional solution of the problem. In the case of the division, extreme weight $W$ values must be located at the boundaries of the problem knowledge granule which are defined by boundary values of variables $\alpha_{V}$ and $\alpha_{V_{s p}}$, Tab. 3. The result of the IPVs division is presented in Fig. 9.

Table 3. Values of the weight $W$ for boundary values of RDMvariables $\alpha_{V}$ and $\alpha_{V_{s p}}$.

\begin{tabular}{c|cccc}
\hline$\alpha_{d}$ & 0 & 0 & 1 & 1 \\
$\alpha_{T}$ & 0 & 1 & 0 & 1 \\
\hline$W$ & 1 & $9 / 11$ & $11 / 9$ & 1 \\
\hline
\end{tabular}

It can be seen from Fig. 9 that minimum weight has the value $\underline{W}=9 / 11$ and maximum: $\bar{W}=11 / 9$. Thus, the result $W \in[9 / 11,11 / 9]$ is consistent with the result obtained with the Moore arithmetic. However, the RDM-arithmetic enables obtaining not only the range [9/11, 11/9] of possible solutions but also the cardinality distribution $\operatorname{card}(x)$ presented in Fig. 10.

Let's consider a problem of the division of same IPVs $[9,11] /[9,11]$ in an another example.

Example 4: Possibility of calculations with fully correlated IPVs (thanks to existence of multiplicative inverse element).

Little Johnny raises money for the piggybank. However he doesn't know how much money he has. He evaluates that the amount $S$ is $[9,11] \$$. Johnny's father promised that he will add into the piggybank the same amount $S$ that is collected, as a reward. After checking the box, the father made his promise. How many dollars did he give as a reward for every dollar collected by Johnny? 


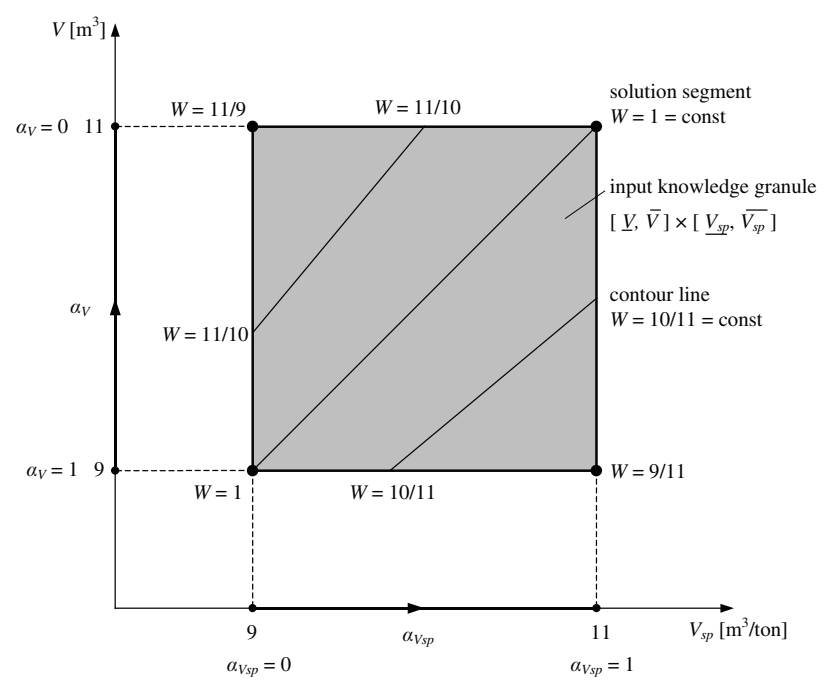

Fig. 9. Illustration of the solution of the same IPVs division problem from Example 3 projected onto the 2D-space $V \times V_{s p}$. Variables $V$ and $V_{s p}$ are completely independent.

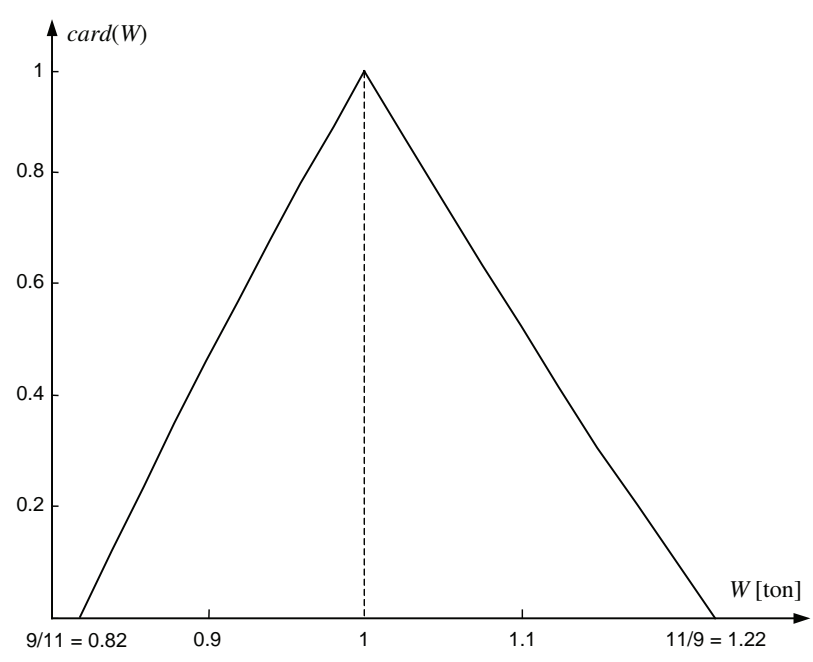

Fig. 10. Normalised cardinality distribution $\operatorname{card}(W)$ of the division of same IPVs $[9,11] /[9,11]$ from Example 3.

For each person the task is very simple. The answer is: for each collected dollar Johnny got 1 dollar as a reward. The correct interval arithmetic should also give the same result which is consistent with the human common sense.

We know that collected amount of money $S$, known only approximately, is included in the interval $S \in[9,11] \$$. The reward $R$ is exactly equal to the amount $S$, so:

$$
R=S, \quad R \in[9,11] \$
$$

The IPVs division can be realised to calculate how great reward falls on each collected dollar. It can be done with the Moore arithmetic:

$$
\left[\underline{R_{1}}, \overline{R_{1}}\right]=\frac{[9,11]}{[9,11]}=[9 / 11,11 / 9] .
$$

It can be seen that the Moore arithmetic is not able to give an exact and obvious answer in this example.

Let's apply the RDM-arithmetic now. The value of the amount $S$ collected by Johnny can be represented with the RDM-variable $\alpha_{S} \in[0,1]$.

$$
S=9+2 \alpha_{S}, \quad \alpha_{S} \in[0,1]
$$

The reward $R$ is exactly equal to the amount $S$ $(R=S)$ so these variables are completely dependent. This equation is a restriction imposed on possible pairs $(R, S)$. Any value the amount $S$ would take, the reward $R$ will be always the same. So, RDM-variables $\alpha_{S}$ and $\alpha_{R}$ must be also equal and it means that only one RDM-variable can be used: $\alpha=\alpha_{S}=\alpha_{R}$.

Now, formulas for variables $S$ and $R$ have form:

$$
\begin{aligned}
& S=9+2 \alpha \\
& R=9+2 \alpha, \quad \alpha \in[0,1] .
\end{aligned}
$$

The division of not exactly known but identical values $S$ and $R$ is given by the formula:

$$
R_{1}=\frac{R}{S}=\frac{9+2 \alpha}{9+2 \alpha}=1, \quad \alpha \in[0,1]
$$

The division result is presented in the 2D-space $R \times S$, Fig. 11. Solution presented in Fig. 11 has the cardinality distribution $\operatorname{card}(x)$ shown in Fig. 12. 


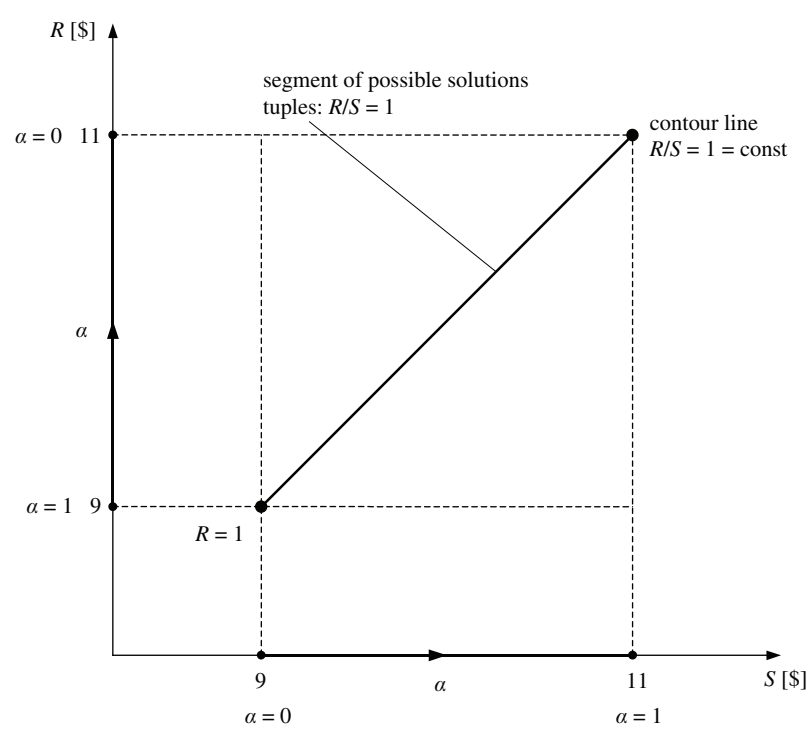

Fig. 11. Illustration of the solution of the same IPVs division problem from Example 4. Variables $V$ and $V_{s p}$ are completely dependent.

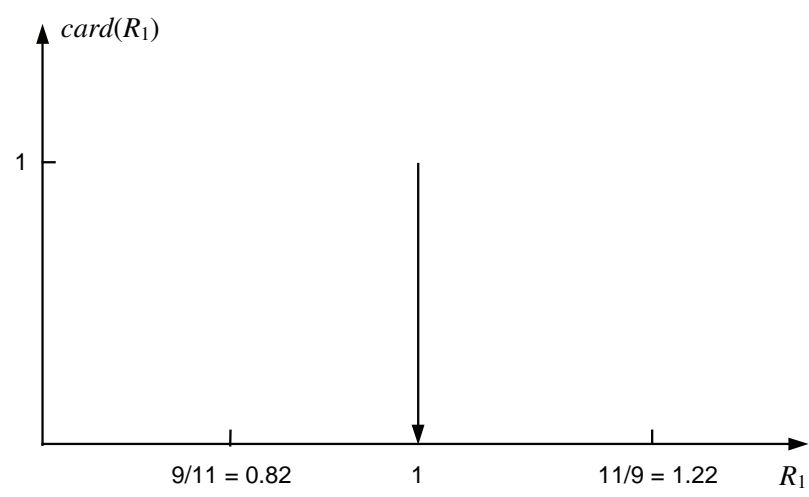

Fig. 12. The cardinality distribution $\operatorname{card}\left(R_{1}\right)$ of the result of the same IPVs division obtained in Example 4.

Now, let's consider another example of the division of same IPVs $[9,11] /[9,11]$.

Example 5: Possibility of calculations with partially correlated IPVs.

A delivery truck transports a material with a specific volume $V_{s p}$ approximately known, $V_{s p} \in[9,11]$ $\mathrm{m}^{3} /$ ton. The value $V_{s p}$ depends on the moisture content of the material. The driver leaves the factory if the truck is loaded at least over the volume $V=9$ $\mathrm{m}^{3}$, if it is at least sufficiently filled up. The truck can carry not more than $11 \mathrm{~m}^{3}$ of a material and the permissible weight of a cargo is 1 ton. How much material $W$ [ton] does vehicle transport?

If the total volume $V$ of the cargo and the specific volume $V_{s p}$ is exactly known then the weight $W$ can be calculated with the idealistic formula:

$$
W=\frac{V}{V_{s p}} .
$$

As, values $V$ and $V_{s p}$ are known only approximately the Moore arithmetic can be applied:

$$
[\underline{W}, \bar{W}]=\frac{[\underline{V}, \bar{V}]}{\underline{\left[V_{s p}, \overline{V_{s p}}\right]}}=\frac{[9,11]}{[9,11]}=\left[\frac{9}{11}, \frac{11}{9}\right] .
$$

Now, let's solve this example with the RDMarithmetic. The approximately known volume value $V$ can be expressed with the variable $\alpha_{V} \in[0,1]$ as a formula:

$$
V=\underline{V}+\alpha_{V}(\underline{V}-\bar{V})=9+2 \alpha_{V}, \quad \alpha_{V} \in[0,1] .
$$

Also $V_{s p}$ can be expressed with the variable $\alpha_{V_{s p}} \in[0,1]$ as a formula:

$V_{s p}=\underline{V_{s p}}+\alpha_{V_{s p}}\left(\underline{V_{s p}}-\overline{V_{s p}}\right)=9+2 \alpha_{V_{s p}}, \alpha_{V_{s p}} \in[0,1]$.

The weight $W$ can be calculated as:

$$
W=\frac{V}{V_{s p}}=\frac{9+2 \alpha_{V}}{9+2 \alpha_{V_{s p}}}, \quad \alpha_{V} \in[0,1], \alpha_{V_{s p}} \in[0,1] .
$$

As the weight $W$ can't be greater than 1 ton we have:

$$
W=\frac{9+2 \alpha_{V}}{9+2 \alpha_{V_{s p}}} \leqslant 1,
$$

and the consequence of the formula (37) is the inequality:

$$
\alpha_{V} \leqslant \alpha_{V_{s p}}
$$

Formula (38) expresses a restriction imposed on pairs $\left(V, V_{s p}\right)$ and correspondingly $\left(\alpha_{V}, \alpha_{V_{s p}}\right)$.

The final, multidimensional problem solution is described by the formula:

$$
\begin{aligned}
& W=\frac{V}{V_{s p}}=\frac{9+2 \alpha_{V}}{9+2 \alpha_{V_{s p}}}, \\
& \alpha_{V} \leqslant \alpha_{V_{s p}}, \alpha_{V} \in[0,1], \alpha_{V_{s p}} \in[0,1] .
\end{aligned}
$$


The set of possible solutions is presented in Fig. 13. The solution granule described by the formula (39) and visualised in Fig. 13 has the cardinality distribution $\operatorname{card}(x)$ presented in Fig. 14.

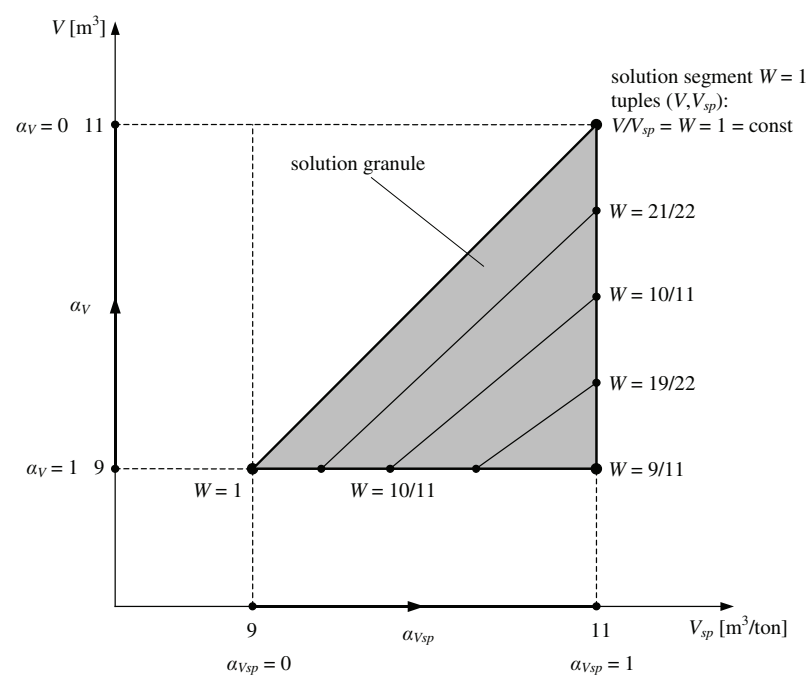

Fig. 13. Visualisation of the solution granule of the same IPVs division from Example 5. Variables $\alpha_{V}$ and $\alpha_{V_{s p}}$ are partially dependent: $\alpha_{V} \leqslant \alpha_{V_{s p}}$.

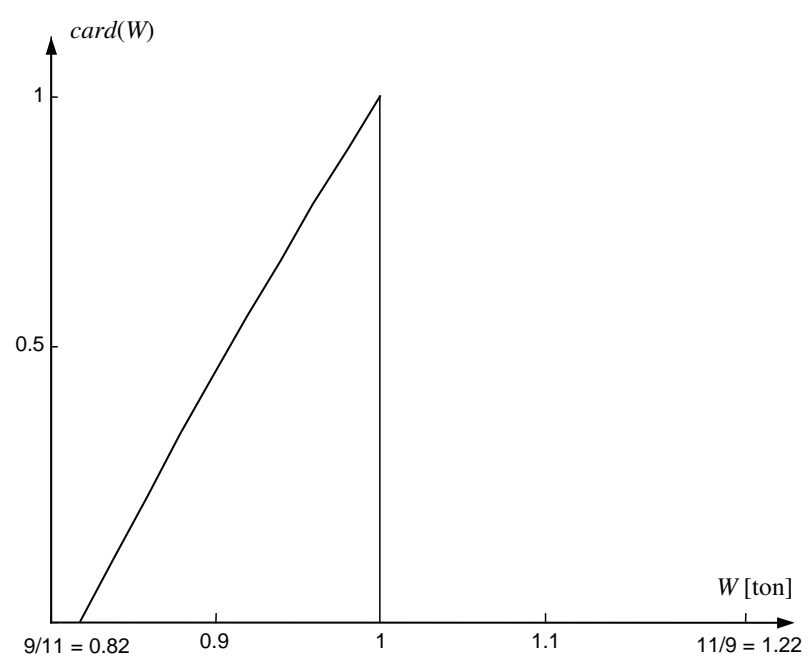

Fig. 14. Cardinality distribution $\operatorname{card}(W)$ of the solution of the same IPVs division from Example 5 as representation of the full solution (39).

It must be noted that the correlation of variables $V$ and $V_{s p}$ from Example 5 described by formulas (37) and (38) was caused by the limited load capac- ity of the truck: $W=V / V_{s p} \leqslant 1 \mathrm{t}$. Such correlation is only one of an infinite number of possible partial dependencies. Theoretically, the maximum load capacity of the truck can have different values, for example: $W \leqslant 1.1 \mathrm{t}, W \leqslant 1.2 \mathrm{t}$, etc. It means that the quotient $[9,11] /[9,11]$ can have an infinite number of solutions.

As a summary, Fig. 15 presents 3 solutions from an infinite number of possible ones obtained in Examples 3, 4 and 5. Solutions were obtained with the application of the RDM-arithmetic (and one for comparison with the Moore arithmetic). Fig. 16 presents cardinality distributions of results of the 'same' IPVs division task obtained in Examples 3, 4 and 5.

As Fig. 15 and Fig. 16 presents, the RDMarithmetic allows for more informative solutions than the Moore arithmetic which loses a lot of information details. The RDM-arithmetic enables for use of the additional knowledge about divided (added, subtracted, multiplied) variables, for example about dependencies between them. It means, that thanks to the RDM-arithmetic we can 'see' much more than thanks to the Moore arithmetic. Divisions of 'identical' IPVs presented in Examples show that we can't apply unified, general formulas for calculations with uncertain data as the Moore arithmetic suggests. Intervals should not be 'anonymous'! Arithmetic operations on IPVs should be carried out taking into account dependencies between variables in a given, particular problem. Thus, there can be infinitely many results of 'identical' intervals division.

\section{Data precisiation}

In the last example, there will be presented the possibility of data precisiation (uncertainty decreasing) of original data items occurring in problems. In this case, a restriction is imposed not only on divided variables $a$ and $b$, but also on the division result $x$. It will be shown, that this restriction allows for greater precisiation of the problem.

\section{Example 6.}

A container of irregular shape contains a material which volume $V\left[\mathrm{~m}^{3}\right]$ was evaluated as $V \in[7,9] \mathrm{m}^{3}$. The material was weighed on an imprecise scales 


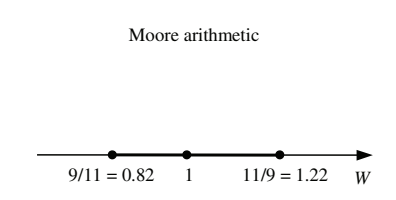

a) Example 3,4,5

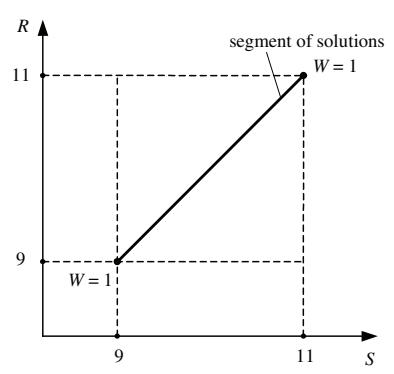

c) Example 4

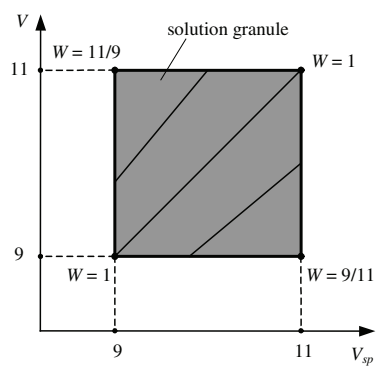

b) Example 3

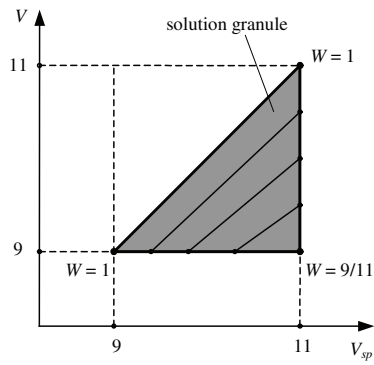

d) Example 5

Fig. 15. Solution granules of the 'same' IPVs division task obtained in Examples 3, 4 and 5 with the Moore arithmetic (a) and with the RDM-arithmetic (b,c,d).
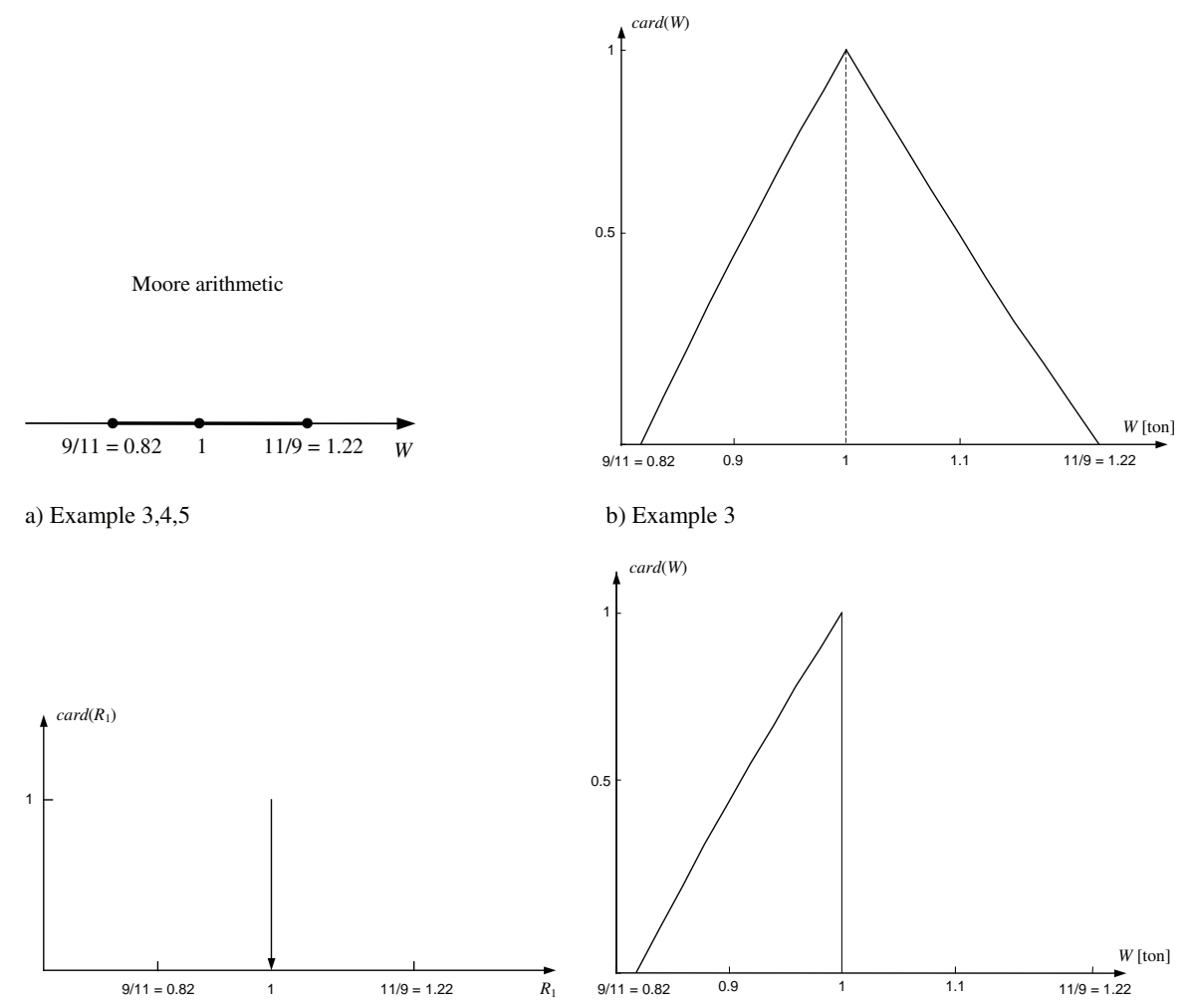

b) Example 3

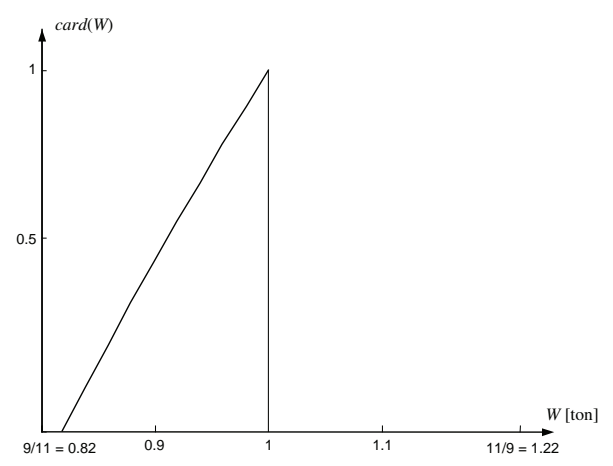

c) Example 4

d) Example 5

Fig. 16. Solution representations of the division of 'identical' IPVs from Examples 3, 4 and 5 with the Moore arithmetic (a) and with the RDM-arithmetic (b,c,d) expressed as cardinality distributions. 
with the result $W \in[6,8]$ tons. It is known from a Material Encyclopedia that this material has a specific volume $V_{s p} \in[0.8,1.0]$ depending on a humidity degree. How can data items be precisiated with IPV-arithmetic?

Particular items are coupled by formula:

$$
V_{s p}=\frac{V}{W}=\frac{[7,9]}{[6,8]}=[0.8,1.0] .
$$

According to the Moore arithmetic, the quotient $V / W$ is equal $[7 / 8,9 / 6]=[0.875,1.5]$. However, it is known from the Encyclopedia that possible domain of $V_{s p}$ equals $[0.8,1.0]$. To investigate this problem, let's express uncertain values of $W, V$, $V_{s p}$ using RDM-variables as: $V=7+2 \alpha_{V}, W=$ $6+2 \alpha_{W}, V_{s p}=0.8+0.2 \alpha_{V_{s p}}, \alpha_{W}, \alpha_{V}, \alpha_{V_{s p}} \in[0,1]$. Then, (40) is transformed into:

$$
\begin{aligned}
& V_{s p}=\frac{V}{W}=\frac{7+2 \alpha_{V}}{6+2 \alpha_{W}}=0.8+0.2 \alpha_{V_{s p}}, \\
& \alpha_{W}, \alpha_{V}, \alpha_{V_{s p}} \in[0,1] .
\end{aligned}
$$

From the contour plot in Fig. 17 we can see that RDM-variable ranges should be reduced:

- $\alpha_{W} \in[0.5,1](\operatorname{not}[0,1])$

- $\alpha_{V} \in[0,0.5](\operatorname{not}[0,1])$.

New ranges can be also easily calculated from (41). Taking into account the lowest values of $V(V=7)$ and the greatest value of $W(W=8)$, we can find the lowest value of $\alpha_{V_{s p}}$. So, the new range:

- $\alpha_{V_{s p}} \in[0.375,1]($ not $[0,1])$.

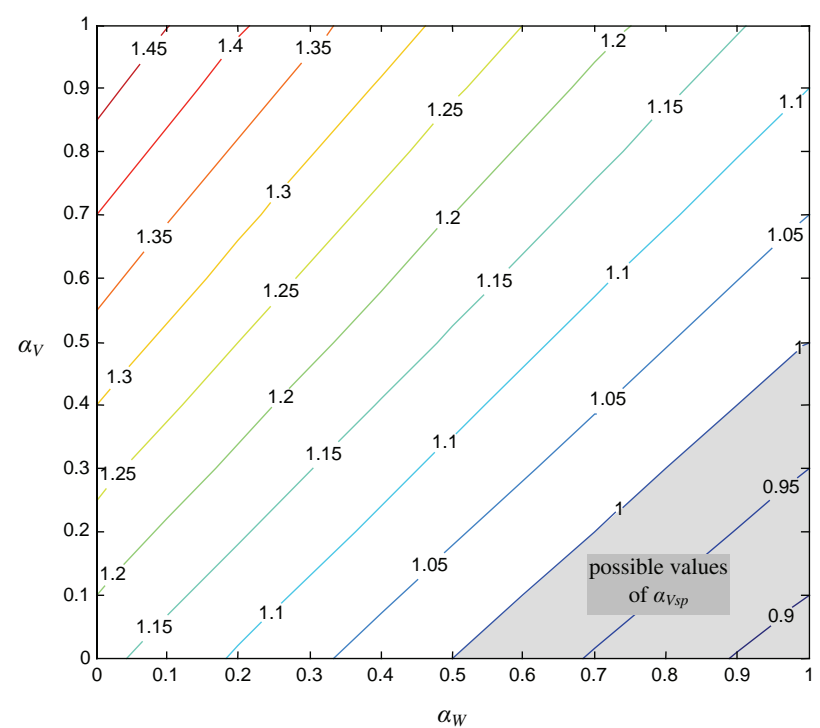

Fig. 17. Contour plot of the function $V_{s p}=f\left(\alpha_{W}, \alpha_{V}\right)$.

The identified possible domains of RDMvariables $\alpha_{V}, \alpha_{W}, \alpha_{V_{s p}}$ correspond to possible domains of weight $W$, volume $V$ and specific volume $V_{s p}$ :

- $W \in[7,8]($ not $[6,8])$,

- $V \in[7,8]($ not $[7,9])$,

- $V_{s p} \in[0.875,1](\operatorname{not}[0.8,1])$,

Fig. 18a shows a cube of the uncertainty of original data items and Fig. 18b shows a triangle of the possible uncertainty achieved thanks to the IPVarithmetic.

Example 6 has shown two interesting things. Firstly, according to the Moore arithmetic, a division result of two IPVs is always the same, e.g. $[7,9] /[6,8]=[7 / 8,9 / 6]=[0.875,1.5]$. However in real life problems, a division result can be different, e.g. $[7,9] /[6,8]=[0.8,1.0]$. This phenomenon is inconsistent with the standard interval arithmetic, but such unintuitive problems can be calculated with the IPV-arithmetic.

Secondly, the IPV-arithmetic based on RDMvariables allows for decreasing of the uncertainty of original data about the problem, because it is able to use dependencies existing between problem variables. As Example 6 has shown, the uncertainty reduction can be considerable. Thus, imprecise mea- 
surements or expert evaluations can be precisiated without additional measurements.
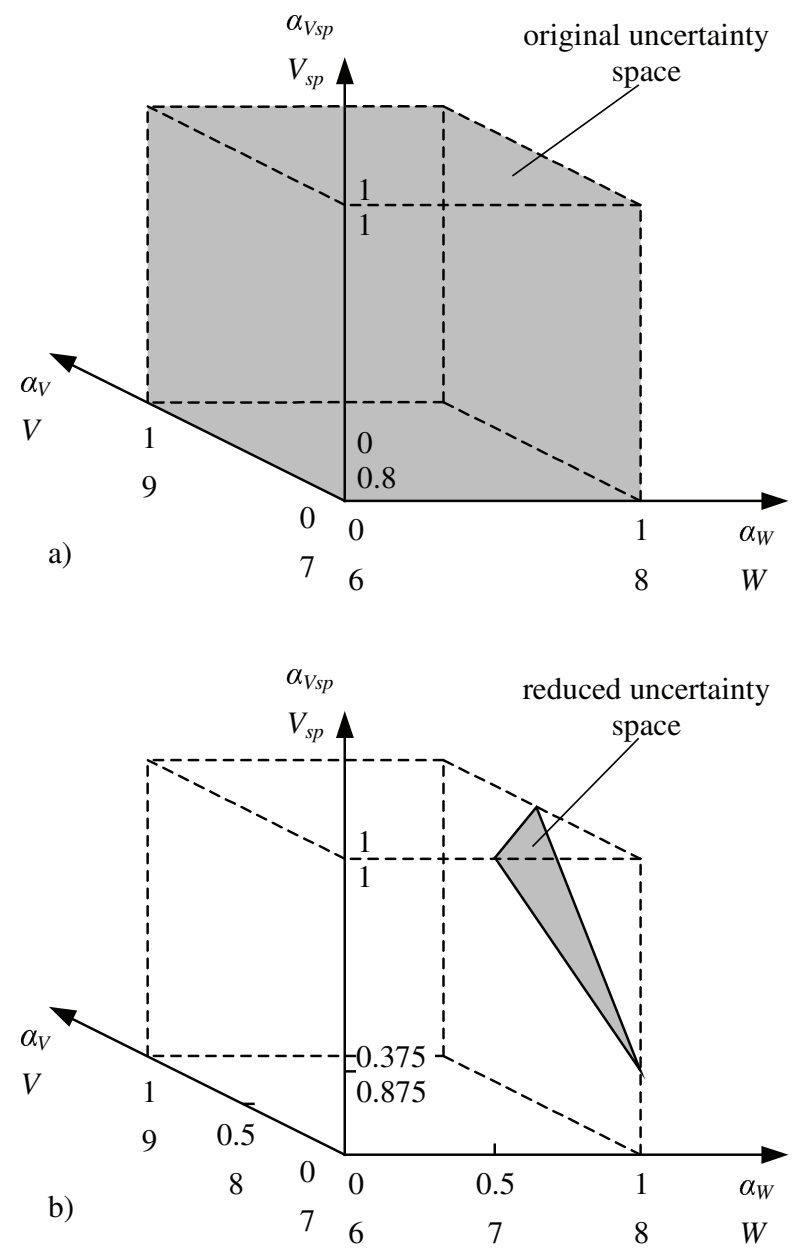

Fig. 18. Visualisation of the original uncertainty space in Example 6 (a) and the reduced space achieved thanks to the IPV-arithmetic (b).

\section{Conclusions}

Real problems are sometimes much more complicated and unintuitive than we can imagine. Their solving requires advanced and effective tools for processing of uncertain data items. An interval is the basic model of an uncertain value and an interval arithmetic is used in higher forms of uncertainty processing, e.g. in the fuzzy arithmetic of type- 1 and type-2, in the intuitionistic fuzzy arithmetic and the probabilistic arithmetic. The standard interval arithmetic is not able to solve more complicated prob- lems.

The paper has presented the IPV-arithmetic, its main mathematical properties and advantages on examples of division operation. All advantages were illustrated by examples of real problems. By introducing the internal RDM-variable $\alpha$ of the interval (which is a model of an uncertain value) it was possible to obtain a deeper knowledge about the problem solution than in the case of application of the Moore arithmetic. The RDM-arithmetic is free from many drawbacks of the Moore arithmetic. It enables solving of complicated problems thanks to possibility of equation transformations, including solving of interval equations. The greatest advantage of the RDM-arithmetic, in Authors opinion, is possibility of taking into account dependencies between intervals and possibility of uncertainty decreasing (very surprising feature), what was illustrated by examples presented in the paper.

The correct solution of more complex mathematical problems, eg. problems described by a system of equations, differential equations or others, requires consideration of dependencies (relationships) existing between the values of the variables in the calculation. The Moore arithmetic does not give us such a possibility so here comes the opportunity for effective application of the RDM-arithmetic described in the paper.

\section{References}

1. R. Moore, "Interval analysis," Prentice Hall, Englewood Cliffs, New Jork (1966).

2. R. Moore, R. Kearfott and M. Cloud, "Introduction to interval analysis," Society for Industrial and Applied Mathematics, Philadelphia (2009).

3. L. Zadeh, "Computing with Words," Springer, Heidelberg, New York (2012).

4. S. Liu and Y. Lin, "Grey systems, theory and applications," Springer Verlag, Berlin, Heidelberg (2010).

5. W. Pedrycz, A. Skowron and V. Kreinovicz (eds), "Handbook of granular computing," J. Wiley, Chichester, England (2008).

6. Y. Yoshimura, "Mathematical model for manoeuvring ship motion (MMG model)," Proceedings of Workshop on Mathematical Models for Operations Involving Ship-Ship Interaction, 1-6, Tokyo (2005).

7. M. Hanss, "Applied fuzzy arithmetic," Springer Verlag, Berlin, Heidelberg (2005). 
8. A. Kaufmann and M. Gupta, "Introduction to fuzzy arithmetic," Van Nostrand Reinhold, New York (1991).

9. A. Piegat, "Fuzzy modeling and control," Physica Verlag, Heidelberg-New York (2001).

10. S. Jaroszewicz and M. Korzeń, "Arithmetic operations on independent random variables: a numerical approach," SIAM Journal of Scientific Computing, 34(3), 1241-1265 (2012).

11. S. Jaroszewicz and M. Korzeń, "Pacal: a Python package for arithmetic computations with random variables," http://pacal.sourceforge.net (2012).

12. R. Williamson, "Probabilistic arithmetic," $\mathrm{PhD}$ thesis, Department of Electrical Engineering, University of Queensland (1989).

13. R. Aliev, W. Pedrycz, B. Fazlollahi, O. Huseynov, A. Alizadeh and B. Guirimov, "Fuzzy logic-based generalized decision theory with imperfect information," Information Sciences, 189, 18-42 (2012).

14. L. Zadeh, "From computing with numbers to computing with words - from manipulation of measurements to manipulation of perceptions," International Journal of Applied Mathematics and Computer Science, 12(3), 307-324 (2002).

15. L. Martinez, D. Ruan and F. Herrera, "Computing with Words in decision support dystems: an overview on models and applications," International Journal of Computational Intelligence Systems, 3(4), 382-395 (2010).

16. J. Liu, L. Martinez, H. Wang, R. Rodriguez and V. Novozhilov, "Computing with Words in risk assessment," International Journal of Computational Intelligence Systems, 3(4), 396-419 (2010).

17. H. Bustince, "Interval-valued fuzzy sets in soft computing," International Journal of Computational Intelligence Systems, 3(2), 215-222 (2010).

18. H. Liu and C. Li, "Fully implicational methods for interval-valued fuzzy reasoning with multi-antecedent rules," International Journal of Computational Intelligence Systems, 4(5),929-945 (2011).

19. R. Gonzalez-del-Campo, L. Garmendia and R. Yager, "Specificity for interval-valued fuzzy sets," International Journal of Computational Intelligence Systems, 5(3), 452-459 (2012).

20. J. Kacprzyk, "Computational intelligence and soft computing: some thoughts on already explored and not yet explored paths," International Journal of Computational Intelligence Systems, 3(2), 223-236 (2010).

21. L. Dymova, "Soft computing in economics and finance," Springer Verlag, Berlin, Heidelberg (2011).

22. A. Sengupta and T. Pal, "Fuzzy preference ordering of interval numbers in decision problems," Springer Verlag, Berlin, Heidelberg (2009).

23. R. Gonzalez-del-Campo, L. Garmendia and J. Re- casens, "Transitive closure of interval-valued fuzzy relations," International Journal of Computational Intelligence Systems, 6(4), 648-657 (2013).

24. X. Zhang, "On interval soft sets with applications," International Journal of Computational Intelligence Systems, 7(1), 186-196 (2014).

25. X. Wang, S. Li and T. Denoeux, "Interval-valued linear model," International Journal of Computational Intelligence Systems, 8(1), 114-127 (2015).

26. F. Meng, C. Tan and X. Chen, "An approach to Atanassov's interval-valued intuitionistic fuzzy multiattribute decision making based on prospect theory," International Journal of Computational Intelligence Systems, 8(3), 591-605 (2015).

27. P. Sevastjanov and L. Dymova, "A new method for solving interval and fuzzy equations: linear case," Information Sciences, 17, 925-937 (2009).

28. P. Sevastjanov, L. Dymova and P. Bartosiewicz, "A framework for rule-base evidential reasoning in the interval settings applied to diagnosing type 2 diabetes," Expert Systems with Applications, 39, 41904200 (2012).

29. A. Piegat and M. Landowski, "Is the conventional interval-arithmetic correct?" Journal of Theoretical and Applied Computer Science, 6(2), 27-44 (2012).

30. A. Piegat and M. Landowski, "Multidimensional approach to interval-uncertainty calculations," New Trends in Fuzzy Sets, Intuitionistic Fuzzy Sets, Generalized Nets and Related Topics, vol. II: Applications, System Research Institute of Polish Academy of Sciences, Warsaw, Poland, 137-152 (2013).

31. A. Piegat and M. Landowski, "Two interpretations of multidimensional RDM arithmetic: multiplication and division," International Journal of Fuzzy Systems, 15(4), 486-496 (2013).

32. A. Piegat and K. Tomaszewska, "Decision-making under uncertainty using Info-Gap theory and a new multidimensional RDM interval arithmetic," Electrical Review, 89(8), 71-76 (2013).

33. A. Piegat and M. Landowski, "Correctness-checking of uncertain-equation solutions on example of the interval-modal method," Modern Approaches in Fuzzy Sets, Intuitionistic Fuzzy Sets, Generalized Nets and Related Topics, vol. 1, eds.: K. Atanassov et al., 159170 (2013).

34. M. Landowski, "Differences between Moore's and RDM interval arithmetic," Proceedings of Thirteenth International Workshop on Intuitionistic Fuzzy Sets and Generalized Nets, Warsaw, Poland (2014).

35. K. Tomaszewska and A. Piegat, "Application of horizontal membership function to the uncertain displacement calculation of a composite massless rod under a tensile load," Soft Computing in Computer and Information Science, 63-72 (2015). 